\title{
Mutational spectrum and associations with clinical features in patients with acute myeloid leukaemia based on next-generation sequencing
}

\author{
YING LI $^{1}$, XIAO LV $^{1}$, XUELING GE $^{1}$, DAI YUAN ${ }^{1}$, MEI DING $^{1}$, CHANGQING ZHEN $^{1}$, WENBO ZHAO $^{1}$, \\ XIN LIU ${ }^{1}$, XIANGHUA WANG ${ }^{1}$, HONGZHI XU ${ }^{1}$, YING LI ${ }^{1,2^{*}}$ and XIN WANG ${ }^{1,2^{*}}$ \\ ${ }^{1}$ Department of Haematology, Shandong Provincial Hospital Affiliated to Shandong University, Jinan, Shandong 250021; \\ ${ }^{2}$ Department of Diagnostics, Shandong University School of Medicine, Jinan, Shandong 250012, P.R. China
}

Received September 14, 2018; Accepted February 19, 2019

DOI: $10.3892 / \mathrm{mmr} .2019 .10081$

\begin{abstract}
The aim of the present study was to examine the associations between 112 acute myeloid leukaemia (AML)-associated genes and the prognosis and clinical features of AML using bioinformatics analysis in 62 patients with AML. A total of 61 gene mutations were identified, and $\geq 1$ mutations were detected in $96.77 \%$ of the patients. A total of 11 frequent mutations were identified, including nucleophosmin 1 (NPM1), Fms related tyrosine kinase 3 (FLT3), DNA methyltransferase $3 \alpha$ (DNMT3A) and Notch 2 (NOTCH2), with a mutation rate of $\geq 10 \%$. The FLT3 mutation was significantly associated with the white blood cell count at the time of diagnosis, and DNMT3A was significantly associated with the French-American-British subtype and cytogenetics of patients with AML. The FLT3, NPMI and $D N M T 3 A$ mutations were significantly associated with a poor overall survival (OS) in patients with AML. In addition, the co-mutation of DNMT3A-CCAAT enhancer binding protein $\alpha(C E B P A)$ was observed to be significantly associated with a poor OS in patients with AML. Furthermore, the functional enrichment analysis revealed that the co-mutations of FLT3-NOTCH2, SETBP1-CREBBP and DNMT3A-CEBPA were significantly enriched in processes of 'negative regulation of cell differentiation' and 'immune system development',
\end{abstract}

Correspondence to: Dr Ying Li (11th author) or Dr Xin Wang, Department of Haematology, Shandong Provincial Hospital Affiliated to Shandong University, 324 Jingwu Weiqi Road, Jinan, Shandong 250021, P.R. China

E-mail: liyjn@126.com

E-mail: xinw@sdu.edu.cn

*Contributed equally

Key words: acute myeloid leukaemia, gene mutation, clinical feature, prognosis, overall survival, DNA methytransferase $3 \alpha$, CCAAT enhancer binding protein $\alpha$ indicating that these mutations may serve crucial roles in the diagnosis and treatment of AML.

\section{Introduction}

Acute myeloid leukaemia (AML) is a heterogeneous group of disorders characterized by the clonal proliferation of progenitor cells or primitive hematopoietic stem cells (1). Due to the development of allogeneic hematopoietic stem cell transplantation (allo-HSCT) for patients with AML, in particular the extensive development of haploidentical allo-HSCT in China, the therapeutic efficacy of treatments for AML have significantly improved throughout previous decades (2). However, the treatment of refractory and relapsed patients remains a significant clinical challenge that has yet to be overcome (3).

AML is typically diagnosed using morphologic, immunologic, cytogenetic and molecular biologic (MICM) classification techniques. However, the accumulation of somatically-acquired genetic changes in hematopoietic progenitor cells serves a vital role in the pathogenesis of AML, including gene mutations, copy number alterations and chromosomal translocation, which provides clinicians with a novel method to diagnose AML (4). Due to the successful application of next generation sequencing (NGS), NGS has become widely used in the analysis of clinical and biological heterogeneity of AML in a clinical setting (5). The study conducted by Corces-Zimmerman et al (6) demonstrated that preleukemic mutation in AML affected the regulation of epigenetic systems, and promoted the survival of hematopoietic stem cells via resistance to chemotherapy. In addition, cyclin D1 and cyclin D2 mutations have been identified to be frequently-occurring events in adult patients with AML at $\mathrm{t}(8 ; 21)(\mathrm{q} 22 ; \mathrm{q} 22)$, and may serve as additional therapeutic targets for AML. Furthermore, the inhibition of mutant isocitrate dehydrogenase $[\mathrm{NADP}(+)] 2$, mitochondrial via AG-221 or DNA methyltransferase activity by 5 -azacytidine has been demonstrated to improve the sensitivity of patients with AML to epigenetic therapy $(7,8)$. These data indicate that mutations in AML exert important functions in the development, treatment and prognosis of AML.

Recently, a spectrum of somatic mutations that were detected by targeted NGS have been identified by Feng et al (9). 
This mutation spectrum contained 112 genes and was based on 121 adult patients with acute leukaemia, and has subsequently been used for the analysis of gene mutations and mutation frequency in malignant hematologic disorders (10). In the present study, amongst the 112-gene mutation panel, a total of 61 gene mutations were determined in the 62 patients with AML. Based on these data, single gene mutations and co-mutations in AML were analysed, followed by the associations with clinical features and the prognosis of AML. The aim of the present study was to provide novel information pertaining to the mechanism of action of AML, with particular emphasis on the roles of co-occurrence gene mutations, in order to provide more efficient therapeutics and to guide the individual course of treatment for patients with AML.

\section{Materials and methods}

Patients and specimen collection. Bone marrow samples were collected from 62 patients with AML (29 males and 33 females, aged between 15-75 years old) who were diagnosed for the first time at Provincial Hospital affiliated to Shandong University (Jinan, China) from January 2016 to December 2016. The diagnosis and categories of AML were performed according to the criteria recommended by the World Health Organization in 2008 (11), and was combined with the MICM characteristics (12). Bone marrow mononuclear cells were isolated by density gradient centrifugation with $2,000 \mathrm{x} \mathrm{g}$ for $15 \mathrm{~min}$ at $4^{\circ} \mathrm{C}$. The present study was approved by the Ethics Committees of Shandong Provincial Hospital and all participants provided written informed consent. The clinical and pathological information of the 62 patients with AML are summarized in Table I.

DNA isolation. For the bone marrow samples, red blood cells were lysed using Red Blood Cell Lysis buffer (Beijing Solarbio Science \& Technology Co., Ltd., Beijing, China). The remaining cells were subsequently counted, and $\sim 1.0 \times 10^{7}$ karyocytes were used to isolate genomic DNA using the Column Blood DNAOUT kit (Tiandz Inc., Beijing, China) according to the manufacturer's protocols.

Detection of gene mutations. A specific target panel for malignant hematologic disorders, which covered hotspots or complete coding regions of 112 genes (Table II) known to be recurrently mutated and/or associated with malignant hematologic disorders was used in the present study (9). A DNA library was constructed using Ion Proton ${ }^{\mathrm{TM}}$ Ion kits (Ion AmpliSeq ${ }^{\mathrm{TM}}$ Library Kit 2.0-96 rxns), according to the manufacturer's protocol (Thermo Fisher Scientific, Inc., Waltham, MA, USA). Subsequent to preparation of the template, the Ion Proton sequencing platform was applied to sequence the exons of these genes using the Ion PI Hi-Q OT2 200 Kit (A26434) and Ion PI Hi-Q Sequencing 200 Kit (A26433). Then, the results were mapped to the National Center for Biotechnology Information hg19 RefSeq with a mean of $>97 \%$ coverage of the targeted regions at an average depth of $800 \mathrm{X}$. The genetic mutation analysis was completed by Ion Reporter system and Variant Reporter software v2.0 (Thermo Fisher Scientific, Inc.). All putative mutations were compared against multiple databases, including dbSNP (13), 1,000 genomes (14), Polyphen-2 (15), and Catalogue of Somatic Mutations In Cancer (16). The detection rate of 5\% mutation frequency was $97-98 \%$.

Statistical analysis of gene mutations. The distribution of detected mutations in the 62 patients was presented using the ggplot2 (version 2.2.1, https://cran.r-project.org/web/packages/ggplot2/) (17) in R software. The mutation frequency of each gene was calculated and the high frequency mutated genes (mutation frequency $>10 \%$ ) were selected for subsequent analysis.

Single gene mutation analysis. Associations between high frequency mutated genes (mutation frequency $>10 \%$ ) and clinical characteristics were analysed using the Pearson's $\chi^{2}$ test (18) in R 3.4.1 software. In addition, high frequency gene mutation profiles were extracted from The Cancer Genome Atlas (TCGA) database (http://cancergenome. nih.gov/). Then, prognosis-associated gene mutations were analysed using Cox univariate regression analysis in a survival package (version 2.40.1; https://cran.r-project. org/package=survival) (19), and the survival results of the high frequency gene mutations were also analysed using Kaplan-Meier survival curves and log-rank tests (20).

Combined gene mutation analysis. Associations between co-mutations with a high frequency and clinical characteristics were analysed using the $1 \mathrm{~m}$ function (https://www.rdocumentation.org/packages/stats/versions/3.4.1/topics/lm) (21) in R 3.4.1 software. The multiple regression model was performed by forced entry linear regression in limma of package $\mathrm{R}$ and bilateral $\mathrm{P}<0.05$ was considered statistically significant. The clinical features that were significantly associated with combined gene mutations were subjected to analysis using the Gene Ontology (GO) $(22,23)$ and the Kyoto Encyclopaedia of Genes and Genomes (KEGG) pathway (24) analyses using Database for Annotation, Visualisation and Integrated Discovery v.6.8 software $(25,26)$ with the threshold of $\mathrm{P}<0.05$, which was considered to indicate a statistically significant difference. In addition, the prognosis-associated co-mutations were analysed using the aforementioned method for single gene mutations.

\section{Results}

Mutations in patients with AML. A total of 61 gene mutations were detected based on the 112 genetic mutations associated with AML Among of the 62 enrolled patients, a total of 60 cases $(96.77 \%)$ presented with at least one mutation, and 52 out of $62(83.87 \%)$ patients exhibited $\geq 2$ mutations. Specifically, 9 cases $(14.52 \%)$ had 2 mutations, 11 patients $(17.74 \%)$ had 3 mutations, 15 patients $(24.19 \%)$ had 4 mutations and 17 patients $(27.42 \%)$ had $>5$ mutations (Fig. 1). Nucleophosmin 1 (NPM1), Fms related tyrosine kinase 3 (FLT3), FAT atypical cadherin 1 (FAT1), ASXL transcriptional regulator $1(A S X L 1)$ and DNA methytransferase $3 \alpha$ (DNMT3A) were the 5 most frequently identified mutations in patients with AML. Using a cut-off frequency of $>10 \%$, a total of 11 high frequency mutations were screened, including NPM1 (22.58\%), FLT3 (22.58\%), FAT1 (20.97\%), ASXL1 (17.74\%), DNMT3A (16.13\%), Notch 2 (NOTCH2; 14.52\%), SET 
Table I. Clinical and pathological information of 62 patients with AML.

\begin{tabular}{lc}
\hline Characteristics & Mean \\
\hline Age at study entry, years (range) & $43.32(15-75)$ \\
Sex & - \\
Male & - \\
Female & \\
WBC count at diagnosis (range) & $31.35(0.80-280.70)$ \\
WBC $\left(10^{9} / 1\right)$ & $63.22(5.83-99.00)$
\end{tabular}

AML FAB subtype

AML with minimal maturation (M0)

$0 / 62$

AML without maturation (M1)

AML with maturation (M2)

Acute myelomonocytic leukemia (M4)

$12 / 62$

Acute monoblastic or monocytic leukemia (M5)

$11 / 62$

Acute erythroid leukemia (M6)

Acute megakaryoblastic leukemia (M7)

Unclassified

Immunophenotype

CD13

CD15

$39 / 62$

CD33

$60 / 62$

CD34

$48 / 62$

CD117

$58 / 62$

MPO

$39 / 62$

CD64

$40 / 62$

HLA-DR

CD56

CD38

$61 / 62$

Cytogenetics

Abnormal karyotype

Normal karyotype

$22 / 62$

Information missing

Risk

High

$12 / 62$

Medium

$31 / 62$

Low

Information missing

$10 / 62$

Induction therapy

IA

DA

Others

Information missing

Response evaluation

Achieving CR

NR

Unevaluated

Information missing 
Table I. Continued.

\begin{tabular}{lcr}
\hline Characteristics & Mean & $\mathrm{N}$ \\
\hline Consolidation therapy after CR & - & $30 / 62$ \\
Chemotherapy & - & $14 / 62$ \\
HSCT & - & $18 / 62$ \\
Information missing & - &
\end{tabular}

AML, acute myeloidleukaemia;WBC, white bloodcell count; FAB, French American British; CR, complete remission; IA, idarubicin + cytarabine . DA, daunorubicin + cytarabine. HSCT, hematopoietic stem cell transplantation; CD13, aminopeptidase N; CD15, sialyl Lewis ${ }^{\mathrm{X}}$; CD33, myeloid cell surface antigen; CD34, hematopoietic progenitor cell antigen CD34; CD117, mast/stem cell growth factor receptor Kit; MPO, myeloperoxidase; CD64, high affinity immunoglobulin gamma Fc receptor I; HLA-DR, human leukocyte antigen-DR isotype; CD56, neural cell adhesion molecule 1; CD38, ADP-ribosyl cyclase/cyclic ADP-ribose hydrolase 1; CR, complete response; NR, non-remission; HSCT, hematopoietic stem cell transplantation.

Table II. Genes closely associated with diseases of the blood system.

\begin{tabular}{rlrllllllllllc}
\hline No. & $\begin{array}{l}\text { Gene } \\
\text { name }\end{array}$ & No. & $\begin{array}{l}\text { Gene } \\
\text { name }\end{array}$ & No. & $\begin{array}{l}\text { Gene } \\
\text { name }\end{array}$ & No. & $\begin{array}{l}\text { Gene } \\
\text { name }\end{array}$ & No. & $\begin{array}{l}\text { Gene } \\
\text { name }\end{array}$ & No. & $\begin{array}{l}\text { Gene } \\
\text { name }\end{array}$ & $\begin{array}{c}\text { Gene } \\
\text { nome }\end{array}$ \\
\hline 1 & ABL1 & 18 & MYC & 35 & SRSF2 & 52 & NF1 & 69 & CCND1 & 86 & PTPN11 & 103 & CSF3R \\
2 & BRAF & 19 & ABCB1 & 36 & BIRC3 & 53 & MAPK1 & 70 & CEBPA & 87 & STX11 & 104 & EZH2 \\
3 & CUX1 & 20 & SF1 & 37 & CBL & 54 & ZRSR2 & 71 & EP300 & 88 & U2AF2 & 105 & IDH2 \\
4 & FANCA & 21 & MAFB & 38 & DNM2 & 55 & IKZF1 & 72 & GATA2 & 89 & CRLF2 & 106 & MAF \\
5 & IL7R & 22 & PRPF40B & 39 & FAT1 & 56 & TET2 & 73 & KIT & 90 & TRAF3 & 107 & PAX5 \\
6 & MPL & 23 & ECT2L & 40 & JAK1 & 57 & TAL1 & 74 & NOTCH2 & 91 & BCL6 & 108 & RB1 \\
7 & PDGFRB & 24 & WT1 & 41 & MYH11 & 58 & ATM & 75 & PTEN & 92 & CREBBP & 109 & SMC3 \\
8 & XPO1 & 25 & ALAS2 & 42 & PRF1 & 59 & CDKN1A & 76 & ARID1A & 93 & ETV6 & 110 & SF3B1 \\
9 & ZMYM3 & 26 & RUNX1 & 43 & KMT2D & 60 & EGFR & 77 & ADAMTS13 & 94 & IDH1 & 111 & ASXL1 \\
10 & SUZ12 & 27 & DDX3X & 44 & SF3A1 & 61 & FLT3 & 78 & FBXW7 & 95 & SH2D1A & 112 & WAS \\
11 & UNC13D & 28 & FANCG & 45 & SETBP1 & 62 & JAK3 & 79 & TP53 & 96 & NRAS & - & - \\
12 & WHSC1 & 29 & ITK & 46 & STXBP2 & 63 & NOTCH1 & 80 & BCL2 & 97 & RAB27A & - & - \\
13 & AKT1 & 30 & MYD8 & 47 & XIAP & 64 & RELN & 81 & LYST & 98 & EED & - & - \\
14 & CALR & 31 & PIK3CA & 48 & CCND3 & 65 & SMC1A & 82 & EPHA7 & 99 & DIS3 & - & - \\
15 & CYLD & 32 & CXCR4 & 49 & DNMT3A & 66 & PRMT5 & 83 & GATA3 & 100 & PHF6 & - & - \\
16 & FANCC & 33 & SH2B3 & 50 & FGFR3 & 67 & FAM46C & 84 & KRAS & 101 & U2AF1 & - & - \\
17 & MUM1 & 34 & SAMHD1 & 51 & JAK2 & 68 & TNFAIP3 & 85 & NPM1 & 102 & PRDM1 & - & - \\
\hline
\end{tabular}

binding protein 1 (SETBP1; 14.52\%), NRAS proto-oncogene, GTPase (NRAS; 14.52\%), CCAAT enhancer binding protein $\alpha$ (CEBPA; 14.52\%), Tet methylcytosine dioxygenase $2(14.52 \%)$ and cyclic adenosine 5'-phosphate response element-binding protein binding protein (CREBBP; 14.52\%) (Fig. 2A). The distribution of high frequency mutations in clinical characteristics are presented in Fig. 2B. The frequencies and types of variants of the 11 high frequency mutations are presented in Table III.

Single mutation analysis. In order to examine the significance of acquired genetic mutations in the development of AML, the present study initially analysed the association between single mutations and clinical features, including white blood cell (WBC) count at diagnosis, French-American-British (FAB) subtype (27), and karyotype using Pearson's $\chi^{2}$ test. As a result,
FLT3, NRAS and CEBPA mutations were significantly associated with WBC count, while ASXL1 and DNMT3A mutations were significantly associated with the FAB subtypes. The DNMT3A mutation was also significantly associated with the variation of the karyotype (Table IV). The survival information of 11 high frequency mutations in AML was extracted from TCGA database, and survival prognosis analysis was performed. The results revealed that 3 single mutations were identified to be negatively associated with a poor overall survival (OS) in patients with AML, including FLT3, NPM1 and DMT3A (Fig. 3).

Combined mutation analysis. The mutation analysis revealed that $56.45 \%$ of patients $(35 / 62)$ exhibited $>2$ high frequency mutations (Fig. 4), indicating that co-occurrence gene mutations were a common phenomenon in AML. The present study 


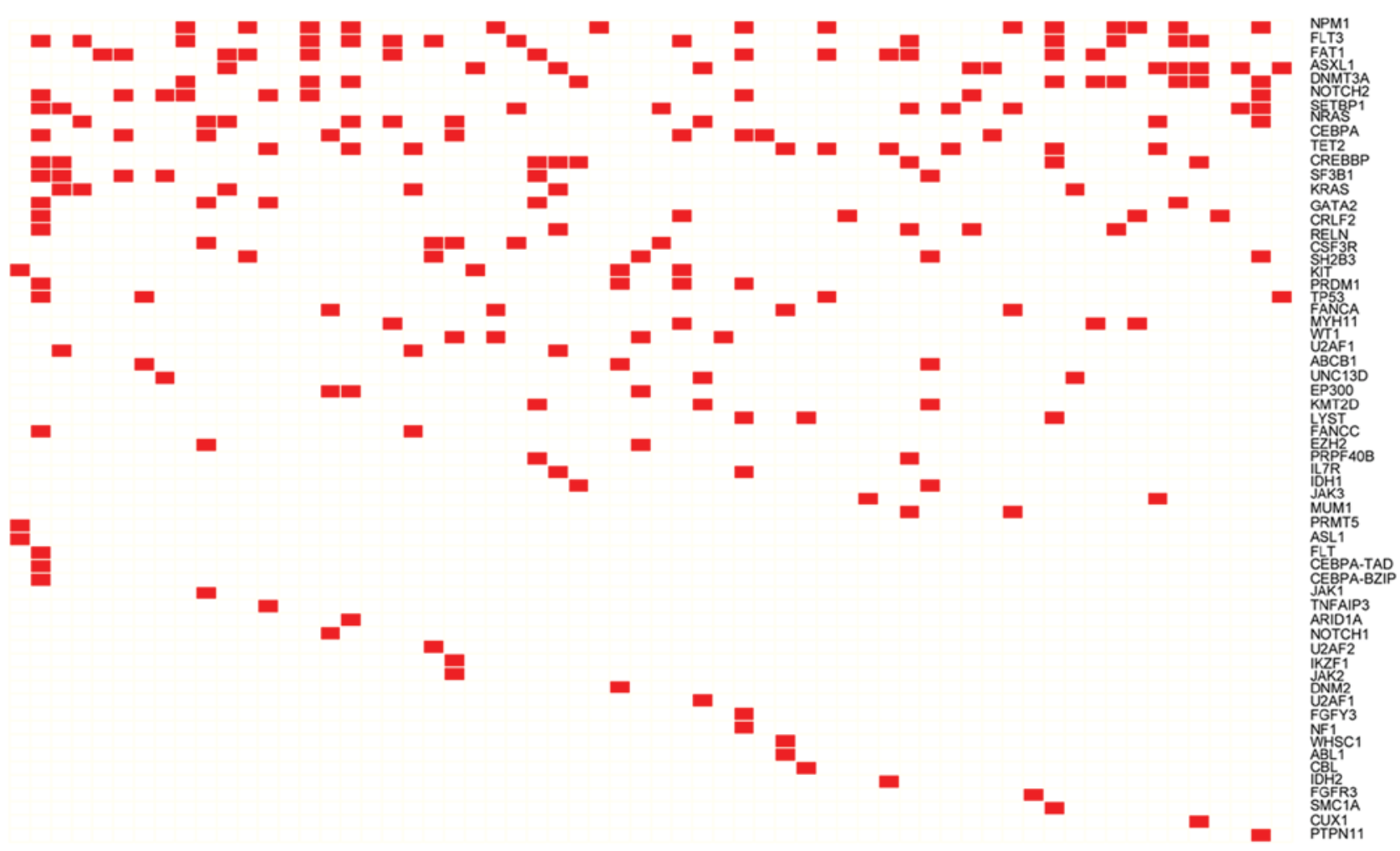

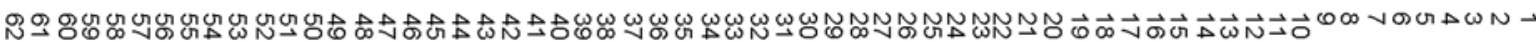

Figure 1. Mutations of 61 genes detected in 62 patients with acute myeloid leukaemia. The x-axis represents the sample number, and the y-axis represents the mutated genes. The red colour indicates the presence of a mutation.

A

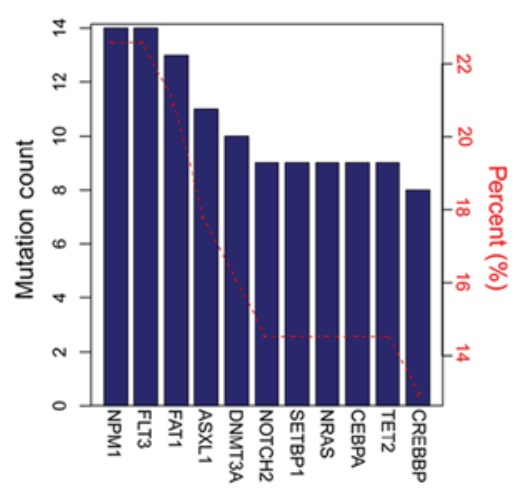

B Karyotype

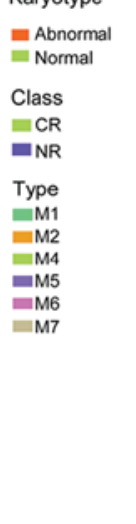

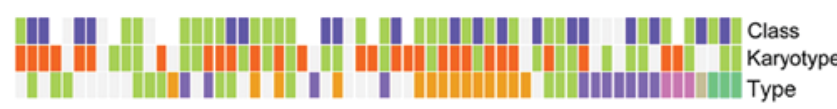

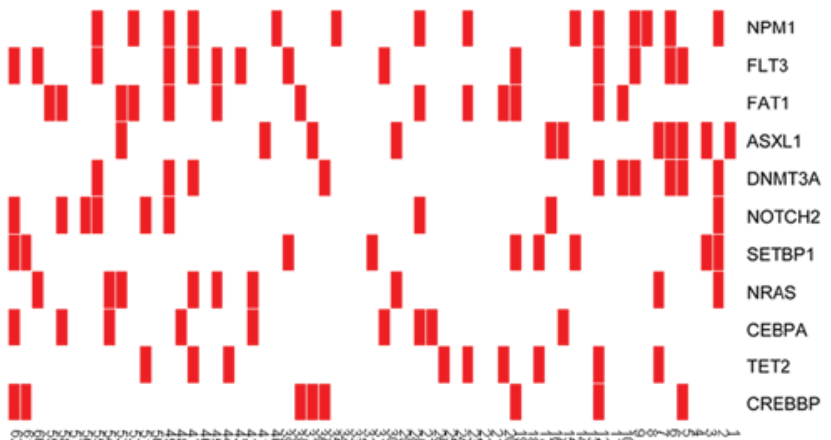

Figure 2. High frequency mutations among 62 patients with acute myeloid leukaemia. (A) Genes whose mutation rates were $>10 \%$. The $\mathrm{x}$-axis represents the gene names, and the y-axis represents the number of patients with mutations in these genes. (B) Distributions of gene mutations. The red colour indicates the presence of a mutation. CR, complete remission; NR, non-remission.

subsequently analysed the association between co-mutations of 11 high frequency mutations and clinical features, including age at the time of diagnosis, sex, bone marrow blast proportion, FAB subtype, karyotype and first course therapeutic response using a multiple regression model. Consequently, a total of 3 combined mutations were identified to be markedly associated with the clinical features of AML. Specifically, the combined mutations FLT3-NOTCH2 and DNMT3A-CEBPA were significantly associated with $\mathrm{WBC}$ and cytogenetics, respectively, while the $S E T B P 1-C R E B B P$ combined mutation was significantly associated with response evaluation and consolidation therapy following complete remission (CR) in AML (Table V). According to the TCGA, among these 3 significant co-mutations, only DNMT3A-CEBPA was significantly associated with a poor OS in patients with AML, and no significant difference was identified in the co-mutation of FLT3-NOTCH2 due to the small sample size (Fig. 5). However, no information about the co-mutation of SETBPI-CREBBP was available in TCGA database; therefore, the present study did not analyse the association between prognosis and the co-mutation SETBPI-CREBBP in patients with AML. 
Table III. Frequencies and types of variants of 11 high frequency mutations.

\begin{tabular}{|c|c|c|c|c|c|}
\hline \multirow{2}{*}{\multicolumn{3}{|c|}{ frequency mutations. }} & \\
\hline & & & Mutated oenes & & \\
\hline \multirow{2}{*}{$\begin{array}{l}\text { Mutated genes } \\
\text { (sample number) }\end{array}$} & \multirow{2}{*}{$\begin{array}{c}\text { Type of variant } \\
\text { (Mutant amino acid) }\end{array}$} & \multirow{2}{*}{ Frequency, $\%$} & (sample number) & (Mutant amino acid) & Frequency, $\%$ \\
\hline & & & \multirow{9}{*}{ NOTCH2 (15) } & p.I1689F & 47.85 \\
\hline \multirow[t]{11}{*}{ NPM1 (11) } & p.W288fs & $>10.00$ & & p.I1689F & 48.99 \\
\hline & p.W288fs & $>10.00$ & & p.1689F & 50.89 \\
\hline & p.W288fs & $>10.00$ & & p.I1789F & 51.70 \\
\hline & p.W288fsX12 & $>10.00$ & & p.1689F & 48.41 \\
\hline & p.W288fs & $>10.00$ & & p.1689F & 48.94 \\
\hline & p.K193R & 5.00 & & p.I1689F & 50.12 \\
\hline & p.E245Q & 45.02 & & p.I1689F & 51.42 \\
\hline & p.W288fs & $>10.00$ & & p.I1689F & 49.20 \\
\hline & p.W288fs & $>10.00$ & \multirow[t]{9}{*}{ SETBP1(15) } & p.P1563L & 20.00 \\
\hline & p.K193R & 4.80 & & p.D868N & 1.65 \\
\hline & p.W288Cfs & $>10.00$ & & p.E1466D & 51.09 \\
\hline \multirow[t]{7}{*}{ FLT3 (23) } & p.D835 $\gamma$ & 15.47 & & p.A1193T & 65.89 \\
\hline & p.V491L & 32.25 & & p.E1466D & 54.58 \\
\hline & ITD & $>10.00$ & & p.E1466D & 52.11 \\
\hline & p.A680V & 9.73 & & p.E1466D & 47.83 \\
\hline & p.D835 & 41.16 & & p.R627C & 51.31 \\
\hline & p.836_837del & 44.29 & & p.R627C & 46.66 \\
\hline & ITD & + & \multirow[t]{12}{*}{ NRAS (15) } & p.G12D & 1.80 \\
\hline \multirow[t]{11}{*}{ FAT1 (21) } & p.V2089I & 54.45 & & p.G12D & 4.74 \\
\hline & p.A4551G & 49.27 & & p.G12C & 4.03 \\
\hline & p.L2822P & 52.81 & & p.G12D & 6.61 \\
\hline & p.V5911 & 50.91 & & p.Q61R & 1.80 \\
\hline & p.A4551G & 48.07 & & p.G13D & 4.85 \\
\hline & p.R1257q & 46.51 & & p.G12D & 30.81 \\
\hline & p.Q587K & 9.41 & & $\begin{array}{l}\text { p.U12D } \\
\text { p.G12D }\end{array}$ & $\begin{array}{l}30.81 \\
46.83\end{array}$ \\
\hline & p.A4551G & 48.14 & & $\begin{array}{l}\text { p.G12D } \\
\text { p.G12D }\end{array}$ & $\begin{array}{r}40.83 \\
175\end{array}$ \\
\hline & p.Q587K & 7.75 & & p.G12D & $\begin{array}{l}1.15 \\
636\end{array}$ \\
\hline & p.Y4232C & 52.24 & & p.G13V & 6.36 \\
\hline & p.V3694I & 58.18 & & p.Q16H & 22.49 \\
\hline \multirow{12}{*}{ ASXL1 (18) } & p.G652S & 50.30 & \multirow[t]{8}{*}{ CEBPA (15) } & p.G32fs & 25.35 \\
\hline & p.G652S & 51.03 & & p.K313delinsQK & 59.26 \\
\hline & p.W898X & 22.89 & & p.A66fs & $>10.00$ \\
\hline & p.W898X & 42.25 & & p.A303P & 48.16 \\
\hline & p.C687X & 41.15 & & p.P23fs & 46.12 \\
\hline & p.G652S & $\begin{array}{l}41.15 \\
51.97\end{array}$ & & p.A72LfsX35 & + \\
\hline & p.G652S & 57.19 & & p.L317delinsRL & 48.27 \\
\hline & p.G1954A & 54.18 & & p.P23fs & 2.70 \\
\hline & p.G652S & 54.72 & \multirow[t]{9}{*}{ TET2 (15) } & p.F868L & 51.68 \\
\hline & p.G652S & 58.47 & & p.S1039L & 48.33 \\
\hline & p.G652S & 52.21 & & p.Q1523X & 2.20 \\
\hline & p.G652S & 57.60 & & p.I1762V & 47.20 \\
\hline \multirow[t]{10}{*}{ DNMT3A (16) } & p.R882H & 42.57 & & p.Q324H & 5.88 \\
\hline & p.R882H & 43.58 & & p.R550X & 10.64 \\
\hline & p.R882C & 47.07 & & p.S1039L & 50.94 \\
\hline & p.R882H & 44.92 & & p.R814C & 49.51 \\
\hline & p.V716D & 45.72 & & p.S1039L & 50.76 \\
\hline & p.R882C & 52.86 & \multirow[t]{5}{*}{ CREBBP (13) } & p.R1140Q & 9.29 \\
\hline & p.R882P & 31.13 & & p.R1140Q & 4.17 \\
\hline & p.R882C & 42.50 & & p.V1924M & 41.56 \\
\hline & p.R882H & 49.56 & & p.R1140Q & 5.20 \\
\hline & p.R882C & 47.38 & & p.R1140Q & 4.35 \\
\hline
\end{tabular}

Table III. Continued. 
Table III. Continued.

\begin{tabular}{lcc}
\hline $\begin{array}{l}\text { Mutated genes } \\
\text { (sample number) }\end{array}$ & $\begin{array}{c}\text { Type of variant } \\
\text { (Mutant amino acid) }\end{array}$ & Frequency, \% \\
\hline p.R1140Q & 5.21 \\
p.R1140Q & 5.75 \\
p.R1140Q & 6.96 \\
\hline
\end{tabular}

NPM1, nucleophosmin 1; FLT3, Fms related tyrosine kinase 3; FAT1, FAT atypical cadherin 1; ASXL1, ASXL transcriptional regu-

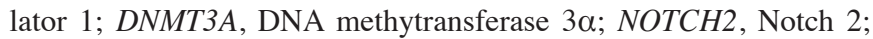
SETBP1, SET binding protein 1; NRAS, NRAS proto-oncogene, GTPase; CEBPA, CCAAT enhancer binding protein $\alpha$; TET2, Tet methylcytosine dioxygenase 2; CREBBP, cyclic adenosine 5 '-phosphate response element-binding protein binding protein.

Table IV.Associations between mutations and clinical features.

A, WBC count at diagnosis

\begin{tabular}{lccc}
\hline & \multicolumn{2}{c}{ WBC (H/L) } & \\
\cline { 2 - 3 } Mutations & Mutation & Non-mutation & P-value \\
\hline FLT3 & $7 / 7$ & $10 / 38$ & 0.04402 \\
NRAS & $6 / 4$ & $11 / 32$ & 0.009661 \\
CEBPA & $5 / 4$ & $12 / 41$ & 0.049879 \\
\hline
\end{tabular}

B, AML FAB subtype

\begin{tabular}{llll}
\hline & \multicolumn{2}{c}{ FAB subtype, } & \\
\cline { 2 - 3 } Mutations & Mutation & Non-mutation & \multirow{2}{*}{ P-value } \\
\cline { 2 - 3 } ASXL1 & $2 / 1 / 3 / 0 / 3 / 0$ & $1 / 13 / 9 / 11 / 0 / 1$ & 0.000115 \\
DNMT3A & $1 / 1 / 0 / 5 / 2 / 0$ & $2 / 13 / 12 / 6 / 1 / 1$ & 0.007636 \\
\hline
\end{tabular}

C, Cytogenetics

\begin{tabular}{|c|c|c|c|}
\hline \multirow[b]{2}{*}{ Mutations } & \multicolumn{2}{|c|}{$\begin{array}{c}\text { Karyotype, } \\
\text { abnormal/normal }\end{array}$} & \multirow[b]{2}{*}{ P-value } \\
\hline & Mutation & Non-mutation & \\
\hline DNMT3A & $2 / 8$ & $26 / 14$ & 0.01446 \\
\hline \multicolumn{4}{|c|}{$\begin{array}{l}\text { WBC, white blood cell; AML, acute myeloid leukaemia; FAB, } \\
\text { French-American-British; FLT3, Fms related tyrosine kinase } 3 \text {; } \\
\text { NRAS, NRAS proto-oncogene, GTPase; CEBPA, CCAAT enhancer } \\
\text { binding protein } \alpha \text {; ASXL1, ASXL transcriptional regulator } 1 \text {; } \\
\text { DNMT3A, DNA methytransferase } 3 \alpha \text {. }\end{array}$} \\
\hline
\end{tabular}

Functional analysis of combined mutations. To additionally investigate the functions of the combined mutations, the 3 co-mutations were subjected to GO and KEGG pathway analyses. The GO analysis revealed that these 3 co-mutations

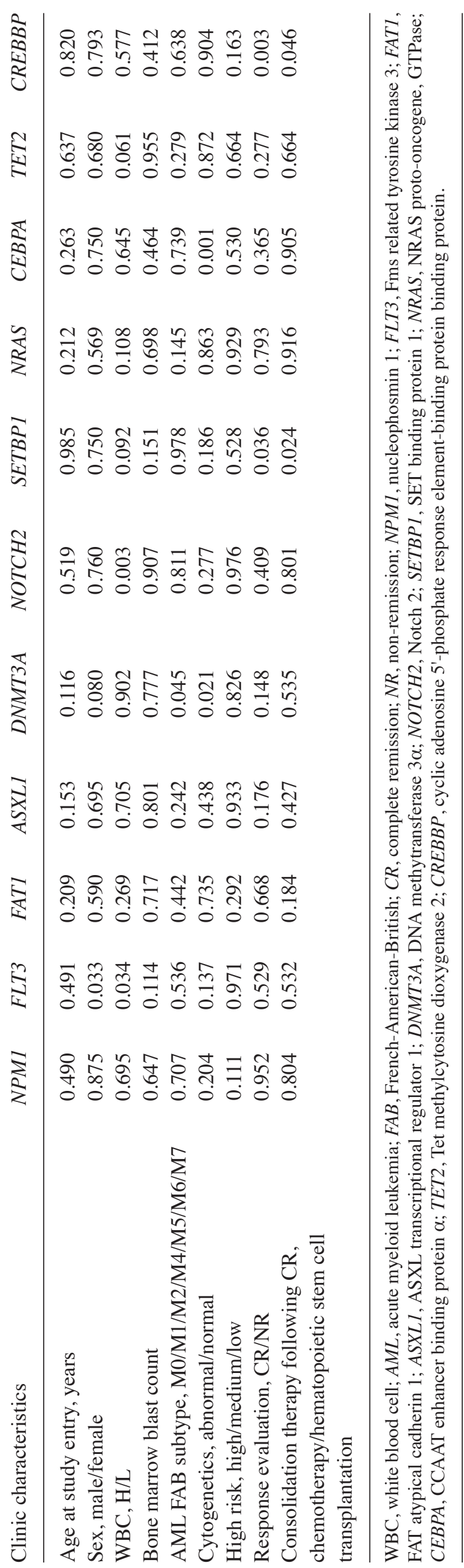


A

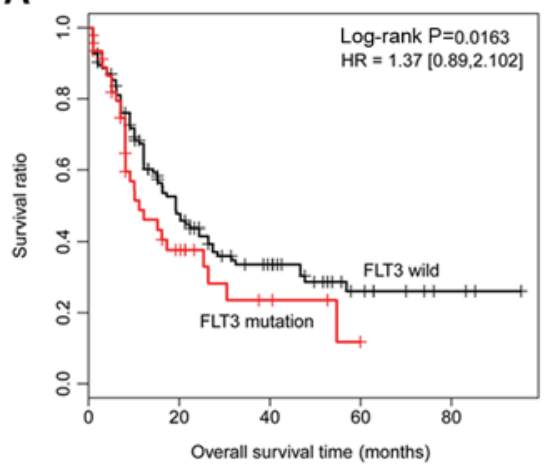

B

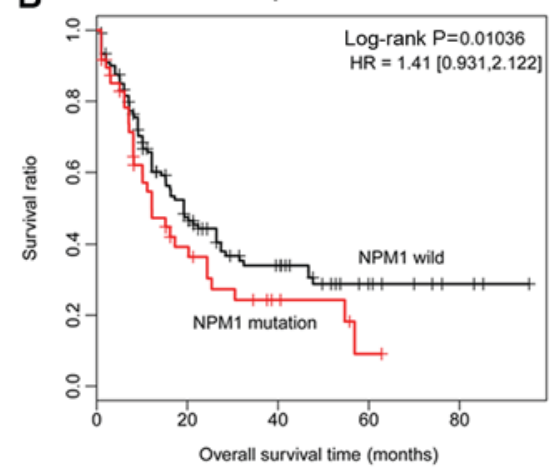

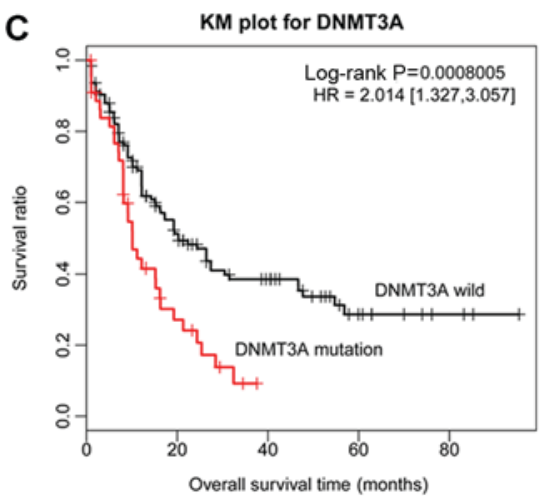

Figure 3. Survival curves of mutated genes based on The Cancer Genome Atlas database. (A) KM survival curve of FLT3. (B) KM survival curve of NPM1. (C) KM survival curve of DNMT3A. KM, Kaplan-Meier; HR, hazard ratio; FLT3, Fms related tyrosine kinase 3; NPM1, nucleophosmin 1; DNMT3A, DNA methytransferase $3 \alpha$.

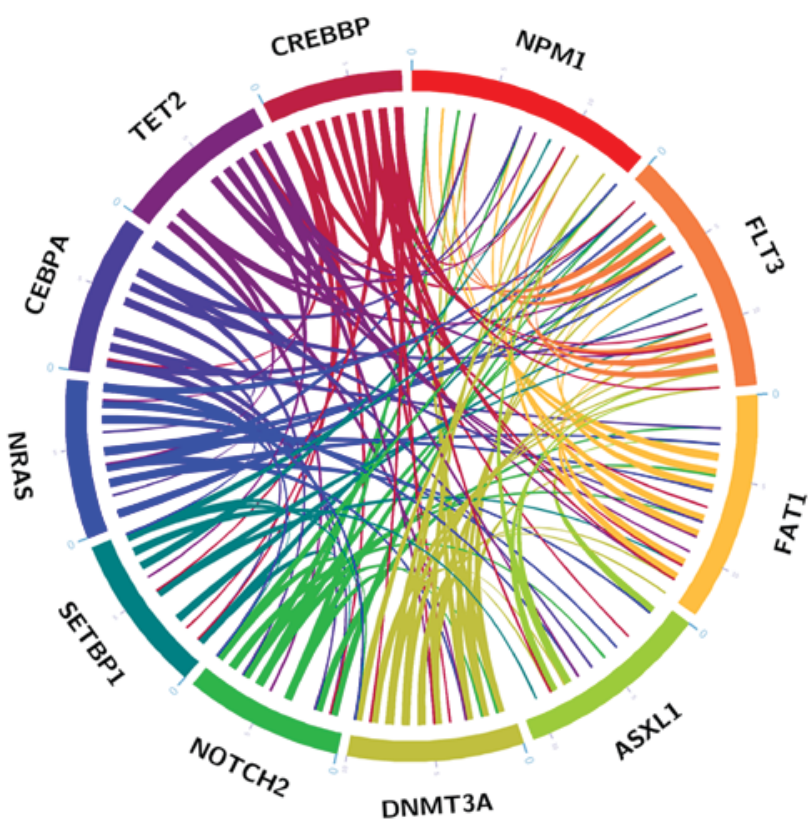

Figure 4. Combined mutations of 11 high frequency mutations. Different colours represent different mutations, and the length of each coloured line represents the mutation number. The curve in the middle of the circle represents the sample, and the two ends of the curve are the genetic mutations that occurred in a sample.

were significantly enriched in 15 biological processes, including 'hemopoietic or lymphoid organ development' $\left(\mathrm{P}=2.15 \times 10^{-3}\right)$, 'negative regulation of cell differentiation' $\left(\mathrm{P}=1.49 \times 10^{-3}\right)$, 'haemopoiesis' $\left(\mathrm{P}=1.78 \times 10^{-3}\right)$ and 'immune system development' $\left(\mathrm{P}=2.42 \times 10^{-3}\right)$. Concomitantly, these 3 co-mutations were also significantly enriched in 3 KEGG pathways, including 'AML' $(\mathrm{P}=0.045)$, 'pathways in cancer' $(\mathrm{P}=0.023)$ and the 'Notch signalling pathway' $(\mathrm{P}=0.036$; Fig. 6).

Analysis of the clinical features of patients with combined mutations. Finally, the present study analysed the common clinical features of patients with these 3 co-mutations. A total of 3 patients with AML were identified to possess the FLT3-NOTCH2 mutation. All of these patients presented with positive aminopeptidase $\mathrm{N}(\mathrm{CD} 13)$, myeloid cell surface antigen
Table VI. Clinical features of 3 patients with concurrent FLT3 and $\mathrm{NOTCH} 2$ mutations.

Sample ID

Clinical features

Sample 2 Sample $9 \quad$ Sample 15

Age, years

Sex

White blood cell $\left(* 10^{9} / 1\right)$

$\begin{array}{ccc}46 & 58 & 67 \\ \text { Female } & \text { Male } & \text { Male } \\ 61.51 & 184.23 & 21.42 \\ 64.5 & 89 & 70 \\ \text { M4 } & \text { Unclassified } & \text { M5 }\end{array}$

Diagnosis

M4

Unclassified

M5

Immunophenotype
CD13
CD15
CD33
CD34
CD117
MPO
CD64
CD56
CD38

HLA-DR

Cytogenetics

Risk

Response evaluation

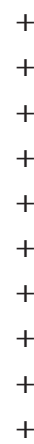

$+\quad+\quad+$

$+\quad+$

$+\quad+\quad+$

$+\quad+$

$+\quad+$

$+\quad+$

$+\quad+\quad+$

$+\quad+\quad+$

$+$

$+\quad+\quad+$

Abnormal Normal Normal

Medium Medium Medium

NR Unevaluated CR

FLT3, Fms related tyrosine kinase 3; NOTCH2, Notch 2; WBC, white blood cell; CD13, aminopeptidase N; CD15, sialyl Lewis ${ }^{\mathrm{x}}$; CD33, myeloid cell surface antigen; CD34, hematopoietic progenitor cell antigen CD34; CD117, mast/stem cell growth factor receptor Kit; MPO, myeloperoxidase; CD64, high affinity immunoglobulin gamma Fc receptor I; HLA-DR, human leukocyte antigen-DR isotype; CD56, neural cell adhesion molecule 1; CD38, ADP-ribosyl cyclase/cyclic ADP-ribose hydrolase 1; NR, non-remission; $\mathrm{CR}$, complete response.

CD33 (CD33), myeloperoxidase (MPO), high affinity immunoglobulin $\gamma$ Fc receptor I (CD64), human leukocyte antigen-DR isotype (HLA-DR) and ADP-ribosyl cyclase/cyclic ADP-ribose hydrolase 1 (CD38) expression (Table VI). In addition, 3 patients 
A

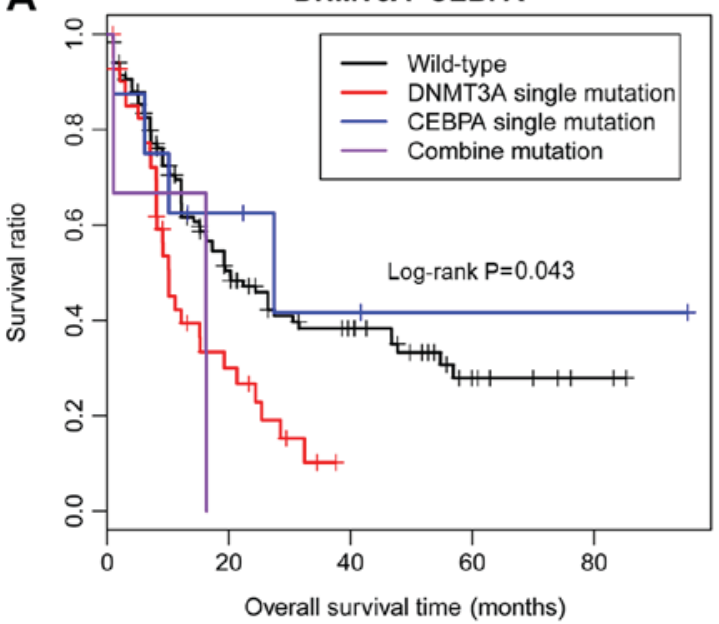

B

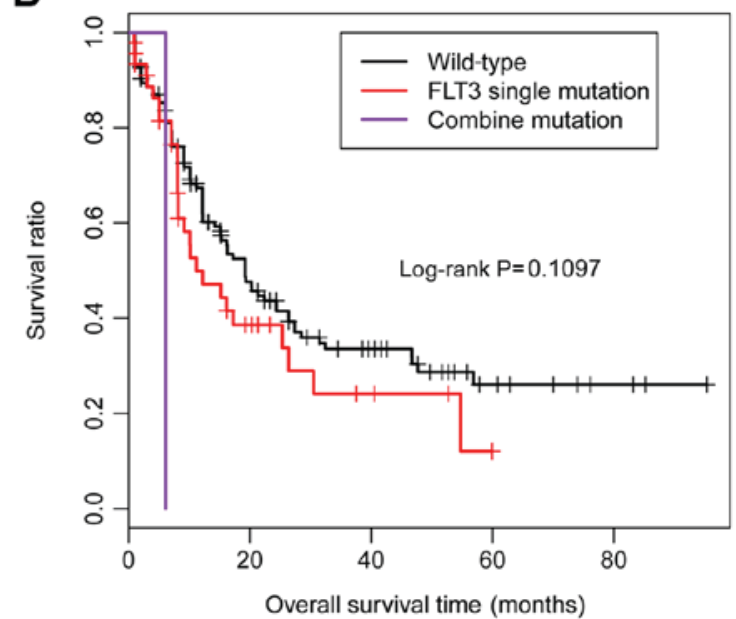

Figure 5. Survival curve analytical results of co-mutations based on The Cancer Genome Atlas database. (A) Kaplan-Meier survival curve of co-mutation of

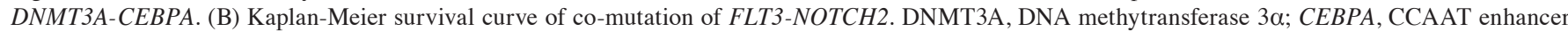
binding protein $\alpha$; FLT3, Fms related tyrosine kinase 3; NOTCH2, Notch 2.

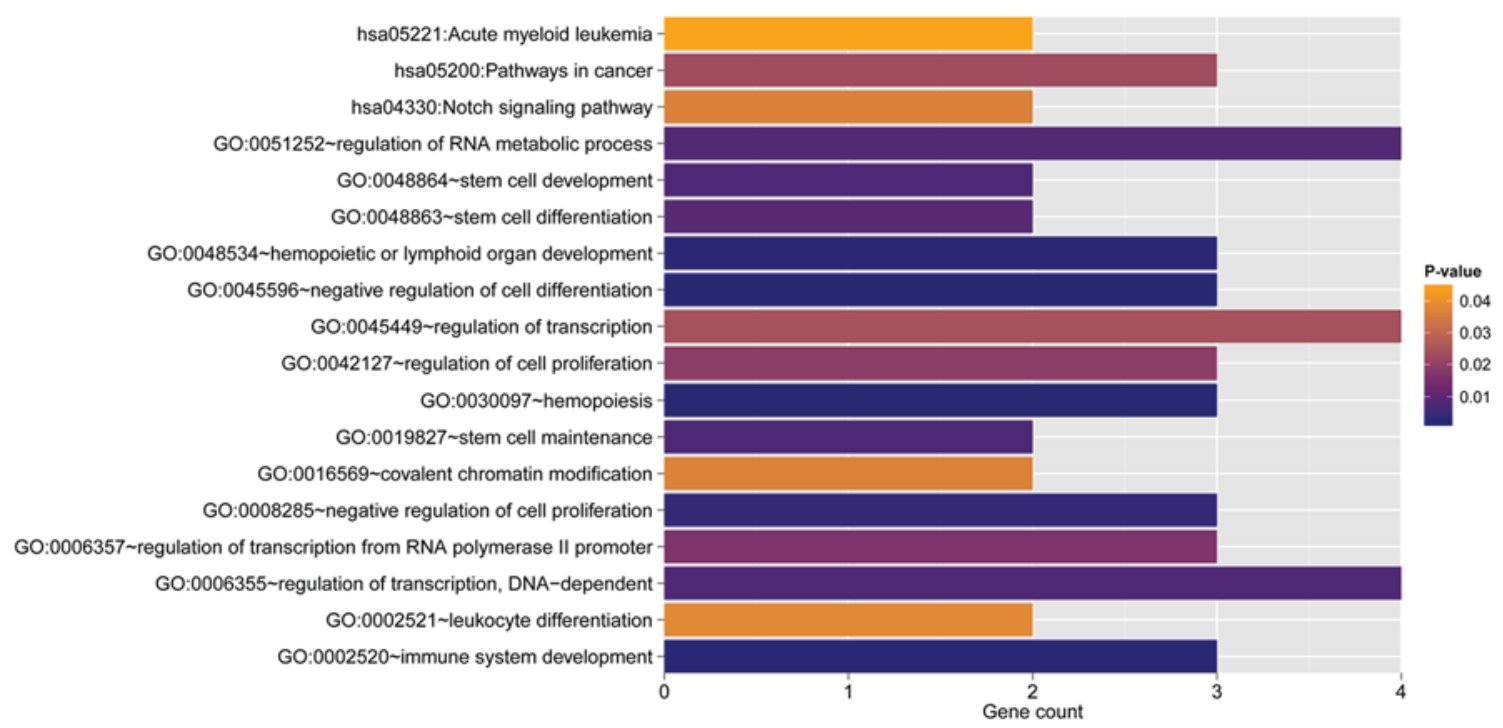

Figure 6. Functional enrichment analysis for co-mutations of Fms related tyrosine kinase 3-Notch 2, DNA methytransferase $3 \alpha$-CCAAT enhancer binding protein $\alpha$ and SET binding protein 1-cyclic adenosine 5'-phosphate response element-binding protein binding protein. GO, gene ontology.

were identified to possess the SETBP-CREBBP mutation, and presented with positive $\mathrm{CD} 13, \mathrm{CD} 33$, hematopoietic progenitor cell antigen CD34 (CD34), mast/stem cell growth factor receptor Kit (CD117), MPO, HLA-DR, neural cell adhesion molecule 1 (CD56) and CD38 expression, and abnormal cytogenetics (Table VII). However, no patients in our study were identified as having the DNMT3A-CEBPA co-mutation.

\section{Discussion}

Molecular abnormalities in multiples genes are involved in the pathogenesis of AML, and have been demonstrated to affect the overall prognosis of AML (28). In the present study, a total of 11 high frequently mutations were identified. Among them, the mutations of FLT3, NRAS, CEBPA, ASXL1 and $D N M T 3 A$ were significantly associated with the clinical features of patients with AML. A total of 3 co-mutations,
FLT3-NOTCH2, DNMT3A-CEBPA and SETBP1-CREBBP, were identified to be significantly associated with the clinical features and prognosis of patients with AML. Functional enrichment analysis demonstrated that mutations in these genes were significantly enriched in the biological process of immune system development, indicating that these combined mutations may serve a critical role in the development of AML.

Genetic mutations are significantly associated with the prognosis and recurrence of AML $(6,29)$. In previous studies, multiple gene mutations have been identified in AML, including FLT3 (30), GATA2 (31), IDH (32) and CPM1 (33). Among these mutations, FLT3, which is the encoding gene of Fms-like receptor tyrosine kinase 3 receptor, is one of the most frequently-occurring mutations detected in AML $(34,35)$. In the present study, the FLT3 mutation was identified in $22.58 \%$ patients; however, this 
Table VII. Clinical features of 3 patients with concurrent $S E T B P 1$ and $C R E B B P$ mutations.

Sample ID

Clinical features

Sample 2 Sample $3 \quad$ Sample 44

\begin{tabular}{lccc}
\hline Age, years & 46 & 23 & 33 \\
Sex & Female & Male & Female \\
WBC $\left(10^{9} / 1\right)$ & 61.51 & 33.72 & 6.23 \\
Bone marrow blast count & 64.5 & 63 & 45 \\
Diagnosis & M4 & Unclassified & M2 \\
Immunophenotype & & & \\
CD13 & + & + & + \\
CD15 & + & + & \\
CD33 & + & + & + \\
CD34 & + & + & + \\
CD117 & + & + & + \\
MPO & + & + & + \\
CD64 & + & - & - \\
HLA-DR & + & + & + \\
CD56 & + & + & + \\
CD38 & + & + & + \\
Cytogenetics & Abnormal & Abnormal & Abnormal \\
Risk & Medium & High & Low \\
Response evaluation & NR & NR & CR \\
\hline
\end{tabular}

SETBP1, SET binding protein 1; CREBBP, cyclic adenosine 5 '-phosphate response element-binding protein binding protein; WBC, white blood cell; CD13, aminopeptidase N; CD15, sialyl Lewis ${ }^{\mathrm{X}}$; CD33, myeloid cell surface antigen; CD34, hematopoietic progenitor cell antigen CD34; CD117, mast/stem cell growth factor receptor Kit; MPO, myeloperoxidase; CD64, high affinity immunoglobulin gamma Fc receptor I; HLA-DR, human leukocyte antigen-DR isotype; CD56, neural cell adhesion molecule 1; CD38, ADP-ribosyl cyclase/cyclic ADP-ribose hydrolase 1; CR, complete response; NR, non-remission.

frequency was decreased compared with the previously described rate $(\sim 30 \%)$ (36). This discrepancy may be due to differences in the ethnicity, geographical region and sample size of the investigated subjects between these studies. In the present study, the FLT3 mutation was also identified to be negatively associated with the survival of patients with AML based on data from TCGA database, which was consistent with the results obtained by a previous study (35). NOTCH2 receptor signalling was demonstrated to govern the differentiation of dendritic cells in the spleen and intestine (37). Additionally, NOTCH2 also controls the rate of generation of long- and short-term repopulating stem cells in mice (38). In the present study, a NOTCH2 mutation was detected in $14.52 \%$ of patients with AML, and the co-mutation of FTL3-NOTCH2 was significantly associated with the variation of WBCs in patients with AML. Additional analysis revealed that patients with the $F L T 3-N O T C H 2$ co-mutation also exhibited positive CD13, CD33, MPO, CD64, HLA-DR and $\mathrm{CD} 38$ expression, indicating that FLT3-NOTCH2 may serve critical roles in the regulation of the immune response.
SETBP1, which is recurrent in myelodysplastic syndromes (MDS) and often co-exists with cytogenetic markers in the progression of AML (39), was also within the top 10 mutations in the present study, with an occurrence of $14.52 \%$. A previous study demonstrated that the SETBP1 mutation was detected in $17 \%$ of patients with secondary AML, which was similar to the results obtained in the present study (40). Cristóbal et al suggested that overexpressed SETBPI predicted an adverse outcome in patients with AML (41). Taken together, these data demonstrate that gene mutations frequently occur in the development of AML and exert crucial functions in regulating the prognosis of AML. In MDS, the SETBPI mutation promotes the leukemic transformation of patients with the ASXL1 mutation (42), indicating that the co-mutation of SETBP1 and ASXL1 may serve a promotive role in the development of AML. Notably, ASXL1 was significantly associated with the FAB subtypes in the present study, suggesting that the SETBPI-ASXL1 mutation was associated with the clinical features of patients with AML. In the present study, the co-mutation of SETBPI and CREBBP was identified in patients with AML, and this was significantly associated with the response evaluation and consolidation therapy following $\mathrm{CR}$, indicating that this co-mutation served an important role in the treatment and prognosis of AML. In addition, the co-mutation of SETBPI and CREBBP consistently presented with abnormal cytogenetics, and positive CD13, CD33, CD34, CD117, CD56, CD38 and MPO expression, indicating that these features may be utilized as potential biomarkers for the diagnosis of patients with AML who present with the SETBPI and CREBBP co-mutation. However, the OS was not significantly different in AML patients with or without mutant SETBP1 and CREBBP; therefore, the underlying mechanism of action requires additional investigation.

DNMT3A is essential for the differentiation of hematopoietic stem cells and its mutations have been identified in $4-22 \%$ of AML cases $(43,44)$. In the present study, the DNMT3A mutation was identified in $16.12 \%$ of patients with AML. The present study also demonstrated that the DNMT3A mutation was significantly associated with the WBC count; however, it was not associated with other mutations in the patient cohort. The DNMT3A mutation was also revealed to be negatively associated with the prognosis of AML, which was consistent with the results obtained by a previous study (45). Although no DNMT3A-CEBPA co-mutation was identified in the present study, the data from TCGA database demonstrated that the co-mutation of DNMT3A-CEBPA was significantly associated with a poor prognosis in patients with AML. Therefore, additional investigations examining the association between the co-mutation of DNMT3A-CEBPA and clinical features should be performed, with a larger patient cohort.

As a result of previous in-depth investigations, several signalling pathways have been demonstrated to be involved in the development and prognosis of AML: The study by Quintás-Cardama et al (46) demonstrated that mutations in the tumor protein p53 pathway are associated with the lowest survival rates in patients with AML. Ufkin et al (47) hypothesized that miR-125a regulated cell proliferation and apoptosis in AML via the ErbB pathway. In the present study, the mutated genes that were significantly associated with 
clinical features were also subjected to functional enrichment analysis. The data revealed that these genes were significantly enriched in the biological processes of 'negative regulation of cell differentiation' and 'immune system development'. Curran et al suggested that targeting the innate immune system may serve as an underlying therapy for AML (48). Additionally, the co-mutations were significantly enriched in the 'Notch signalling pathway'. Takam Kamga et al (49) demonstrated that Notch signalling enhanced bone marrow stromal cell-mediated chemoresistance in AML, and the activation of Notch antagonizes DNA-binding protein Ikaros-based tumor suppression in T-cell ALL (50). These data indicated that these clinical features and mutations of the associated genes may promote the development of ALL via dysregulating the differentiation of hematopoietic cells and the immune response.

In conclusion, FLT3, NOTCH2, and DNMT3A were the 3 mutations with the highest frequencies identified in AML. Specifically, the mutations in FLT3 and DNMT3A were significantly associated with a poor prognosis in patients with AML. In addition, co-mutations of FLT3-NOTCH2 and SETBP1-CREBBP were significantly associated with the clinical features of patients with AML, and may serve a critical role in AML, via regulating the differentiation of hematopoietic cells and the immune response. Genome sequencing is an important method for the detection of mutations in patients with AML, which may provide useful information in understanding the mechanism of AML, which would assist in guiding individual treatment strategies.

\section{Acknowledgements}

Not applicable.

\section{Funding}

The present study received funding from the National Natural Science Foundation (grant nos. 81473486 and 81770210), the Technology Development Projects of Shandong Province (grant nos. 2014GSF118021 and 2017GSF18189), the Taishan Scholar Foundation of Shandong Province and The Key Research and Development Project of Shandong Province, China (grant no. 2015GSF118025).

\section{Availability of data and materials}

The software packages and raw data used to support the results of the present study are available from the corresponding author upon request.

\section{Authors' contributions}

YL (first author), XinW and HX made substantial contributions to the conception and design of the present study, and drafted the manuscript. XLiu, CZ and WZ performed the data acquisition. XG, DY and XLv performed the data analysis and interpretation. YL (11th author), MD and XiaW contributed to the design of the study, and performed the bioinformatic analysis. All authors have read and approved the manuscript.

\section{Ethics approval and consent to participate}

The present study was approved by the Ethics Committees of Shandong Provincial Hospital. All participants provided written informed consent.

\section{Patient consent for publication}

All participants provided written informed consent.

\section{Competing interests}

The authors declare that they have no competing interests.

\section{References}

1. Khwaja A, Bjorkholm M, Gale RE, Levine RL, Jordan CT, Ehninger G,Bloomfield CD, Estey E, Burnett A, Cornelissen JJ, et al: Acute myeloid leukaemia. Nat Rev Dis Primers 2: 16010, 2016.

2. Pan Y, Liu D, Wei Y, Su D, Lu C, Hu Y and Zhou F: Azelaic acid exerts antileukemic activity in acute myeloid leukemia. Front Pharmacol 8: 359, 2017.

3. Liang H, Zheng QL, Fang P, Zhang J, Zhang T, Liu W, Guo M, Robinson CL, Chen SB, Chen XP, et al: Targeting the PI3K/AKT pathway via GLI1 inhibition enhanced the drug sensitivity of acute myeloid leukemia cells. Sci Rep 7: 40361, 2017.

4. Sanders MA and Valk PJ: The evolving molecular genetic landscape in acute myeloid leukaemia. Curr Opin Hematol 20: 79-85, 2013.

5. Kohlmann A, Grossmann V, Nadarajah N and Haferlach T: Next-generation sequencing-feasibility and practicality in haematology. Br J Haematol 160: 736-753, 2013.

6. Corces-Zimmerman MR, Hong WJ, Weissman IL, Medeiros BC and Majeti R: Preleukemic mutations in human acute myeloid leukemia affect epigenetic regulators and persist in remission. Proc Natl Acad Sci USA 111: 2548-2553, 2014.

7. Shih AH, Meydan C, Shank K, Garrett-Bakelman FE, Ward PS, Intlekofer AM, Nazir A, Stein EM, Knapp K, Glass J, et al: Combination targeted therapy to disrupt aberrant oncogenic signaling and reverse epigenetic dysfunction in IDH2- and TET2-mutant acute myeloid leukemia. Cancer Discov 7: 494-505, 2017.

8. Yen K, Travins J, Wang F, David MD, Artin E, Straley K, Padyana A, Gross S, DeLaBarre B, Tobin E, et al: AG-221, a first-in-class therapy targeting acute myeloid leukemia harboring oncogenic IDH2 mutations. Cancer Discov 7: 478-493, 2017.

9. Feng J, Li Y, Jia Y, Fang Q, Gong X, Dong X, Ru K, Li Q, Zhao X, Liu K, et al: Spectrum of somatic mutations detected by targeted next-generation sequencing and their prognostic significance in adult patients with acute lymphoblastic leukemia. J Hematol Oncol 10: 61, 2017.

10. Feng J, Gong XY, Jia YJ, Liu KQ, Li Y, Dong XB, Fang QY, $\mathrm{Ru} \mathrm{K}$, Li QH, Wang HJ, et al: Spectrum of somatic mutations and their prognostic significance in adult patients with B cell acute lymphoblastic leukemia. Zhonghua Xue Ye Xue Za Zhi 39: 98-104, 2018 (In Chinese).

11. Vardiman JW, Thiele J, Arber DA, Brunning RD, Borowitz MJ, Porwit A, Harris NL, Le Beau MM, Hellström-Lindberg E, Tefferi A and Bloomfield CD: The 2008 revision of the World Health Organization (WHO) classification of myeloid neoplasms and acute leukemia: Rationale and important changes. Blood 114: 937-951, 2009.

12. Löffler H: Morphology, immunology, cytochemistry, and cytogenetics and the classification of subtypes in AML. Haematol Blood Transfus 33: 239-242, 1990.

13. Sherry ST, Ward MH, Kholodov M, Baker J, Phan L, Smigielski EM and Sirotkin K: dbSNP: The NCBI database of genetic variation. Nucleic Acids Res 29: 308-311, 2001.

14. 1000 Genomes Project Consortium, Abecasis GR, Auton A, Brooks LD, DePristo MA, Durbin RM, Handsaker RE, Kang HM, Marth GT and McVean GA: An integrated map of genetic variation from 1,092 human genomes. Nature 491: 56-65, 2012. 
15. Adzhubei I, Jordan DM and Sunyaev SR: Predicting functional effect of human missense mutations using PolyPhen-2. Curr Protoc Hum Genet Chapter 7: Unit7.20, 2013.

16. Forbes SA, Bindal N, Bamford S, Cole C, Kok CY, Beare D, Jia M, Shepherd R, Leung K, Menzies A, et al: COSMIC: Mining complete cancer genomes in the catalogue of somatic mutations in cancer. Nucleic Acids Res 39: D945-D950, 2011.

17. Ito K and Murphy D: Application of ggplot2 to pharmacometric graphics. CPT Pharmacometrics Syst Pharmacol 2: e79, 2013.

18. Plackett RL: Karl pearson and the Chi-squared test. Int Stat Rev 51: 59-72, 1983

19. Ritchie ME, Phipson B, Wu D, Hu Y, Law CW, Shi W and Smyth GK: limma powers differential expression analyses for RNA-sequencing and microarray studies. Nucleic Acids Res 43: e47, 2015

20. Goel MK, Khanna P and Kishore J: Understanding survival analysis: Kaplan-Meier estimate. Int J Ayurveda Res 1: 274-278, 2010.

21. Zhang YY,Zhou XB, Wang QZ and Zhu XY: Quality of reporting of multivariable logistic regression models in Chinese clinical medical journals. Medicine (Baltimore) 96: e6972, 2017.

22. Ashburner M, Ball CA, Blake JA, Botstein D, Butler H, Cherry JM, Davis AP, Dolinski K, Dwight SS, Eppig JT, et al: Gene ontology: Tool for the unification of biology. The gene ontology consortium. Nat Genet 25: 25-29, 2000.

23. The Gene Ontology Consortium: The gene ontology resource: 20 years and still GOing strong. Nucleic Acids Res 47: D330-D338, 2019.

24. Kanehisa M, Sato Y, Furumichi M, Morishima K and Tanabe M New approach for understanding genome variations in KEGG. Nucleic Acids Res 47: D590-D595, 2019.

25. Huang da W, Sherman BT and Lempicki RA: Bioinformatics enrichment tools: Paths toward the comprehensive functional analysis of large gene lists. Nucleic Acids Res 37: 1-13, 2009.

26. Huang Da W, Sherman BT and Lempicki RA: Systematic and integrative analysis of large gene lists using DAVID bioinformatics resources. Nat Protoc 4: 44-57, 2009.

27. Bennett JM, Catovsky D, Daniel MT, Flandrin G, Galton DA, Gralnick HR and Sultan C: Proposed revised criteria for the classification of acute myeloid leukemia: A report of the French-American-British Cooperative Group. Ann Intern Med 103: 620-625, 1985.

28. Mukherjee A, Nan X, Ensor J, Randhawa JK, Pingali SRK, Zieske AW, Olsen RJ, Chung B and Iyer SP: An integer weighted genomic mutation score (GMS) using next generation sequencing is predictive of prognosis in intermediate risk AML patients. Blood 130: 3940, 2017.

29. Klco JM, Miller CA, Griffith M, Petti A, Spencer DH, Ketkar-Kulkarni S, Wartman LD, Christopher M, Lamprecht TL, Helton NM, et al: Association between mutation clearance after induction therapy and outcomes in acute myeloid leukemia. JAMA 314: 811-822, 2015.

30. Wander SA, Levis MJ and Fathi AT: The evolving role of FLT3 inhibitors in acute myeloid leukemia: Quizartinib and beyond. Ther Adv Hematol 5: 65-77, 2014.

31. Pasquet M, Bellanné-Chantelot C, Tavitian S, Prade N, Beaupain B, Larochelle O, Petit A, Rohrlich P, Ferrand C, Van Den Neste E, et al: High frequency of GATA2 mutations in patients with mild chronic neutropenia evolving to MonoMac syndrome, myelodysplasia, and acute myeloid leukemia. Blood 121: 822-829, 2013.

32. Im AP, Sehgal AR, Carroll MP, Smith BD, Tefferi A, Johnson DE and Boyiadzis M: DNMT3A and IDH mutations in acute myeloid leukemia and other myeloid malignancies: Associations with prognosis and potential treatment strategies. Leukemia 28 $1774-1783,2014$

33. Etchin J, Sanda T, Mansour MR, Kentsis A, Montero J, Le BT, Christie AL, McCauley D, Rodig SJ, Kauffman M, et al: KPT-330 inhibitor of CRM1 (XPO1)-mediated nuclear export has selective anti-leukaemic activity in preclinical models of T-cell acute lymphoblastic leukaemia and acute myeloid leukaemia. Br J Haematol 161: 117-127, 2013.

34. Höckendorf U, Yabal M and Jost PJ: RIPK3-dependent cell death and inflammasome activation in FLT3-ITD expressing LICs. Oncotarget 7: 57483-57484, 2016.
35. Kurtz SE, Wilmot B, McWeeney S, Vellanki A, Local A, Benbatoul K, Folger P, Sheng S, Zhang H, Howell SB, et al: CG'806, a first-in-class FLT3/BTK inhibitor, exhibits potent activity against AML patient samples with mutant or wild type FLT3, as well as other hematologic malignancy subtypes. Clin Cancer Res 23: 442017.

36. Nishida A, Yuasa M, Kageyama K, Ishiwata K, Takagi S, Yamamoto H, Asano-Mori Y, Yamamoto G, Uchida N, Izutsu $\mathrm{K}$, et al: High disease-free and overall survival rate following allogeneic hematopoietic stem cell transplantation for FLT3-mutated acute myeloid leukemia even in non-remission status. Blood 128: 2283, 2016.

37. Lewis KL, Caton ML, Bogunovic M, Greter M, Grajkowska LT, Ng D, Klinakis A, Charo IF, Jung S, Gommerman JL, et al: Notch2 receptor signaling controls functional differentiation of dendritic cells in the spleen and intestine. Immunity 35: 780-791, 2011.

38. Varnum-Finney B, Halasz LM, Sun M, Gridley T, Radtke F and Bernstein ID: Notch2 governs the rate of generation of mouse long- and short-term repopulating stem cells. J Clin Invest 121: 1207-1216, 2011.

39. Fernandez-Mercado M, Pellagatti A, Di Genua C, Larrayoz MJ, Winkelmann N, Aranaz P, Burns A, Schuh A, Calasanz MJ, Cross NC and Boultwood J: Mutations in SETBP1 are recurrent in myelodysplastic syndromes and often coexist with cytogenetic markers associated with disease progression. Br J Haematol 163: 235-239, 2013

40. Makishima H, Yoshida K, Nguyen N, Przychodzen B, Sanada M, Okuno Y, Ng KP, Gudmundsson KO, Vishwakarma BA, Jerez A, et al: Somatic SETBP1 mutations in myeloid malignancies. Nat Genet 45: 942-946, 2013

41. Cristóbal I, Blanco FJ, Garcia-Orti L, Marcotegui N, Vicente C, Rifon J, Novo FJ, Bandres E, Calasanz MJ, Bernabeu C and Odero MD: SETBP1 overexpression is a novel leukemogenic mechanism that predicts adverse outcome in elderly patients with acute myeloid leukemia. Blood 115: 615-625, 2010

42. Inoue D, Kitaura J, Matsui H, Hou HA, Chou WC, Nagamachi A, Kawabata KC, Togami K, Nagase R, Horikawa S, et al: SETBP1 mutations drive leukemic transformation in ASXL1-mutated MDS. Leukemia 29: 847-857, 2015.

43. Ley TJ, Ding L, Walter MJ, McLellan MD, Lamprecht T, Larson DE, Kandoth C, Payton JE, Baty J, Welch J, et al: DNMT3A mutations in acute myeloid leukemia. N Engl J Med 363: 2424-2433, 2010

44. Abdel-Wahab O, Pardanani A, Rampal R, Lasho TL, Levine RL and Tefferi A: DNMT3A mutational analysis in primary myelofibrosis, chronic myelomonocytic leukemia and advanced phases of myeloproliferative neoplasms. Leukemia 25: 1219-1220, 2011.

45. Kao HW, Liang DC, Kuo MC, Wu JH, Dunn P, Wang PN, Lin TL, Shih YS, Liang ST, Lin TH, et al: High frequency of additional gene mutations in acute myeloid leukemia with MLL partial tandem duplication: DNMT3A mutation is associated with poor prognosis. Oncotarget 6: 33217-33225, 2015.

46. Quintás-Cardama A, Hu C, Qutub A, Qiu YH, Zhang X, Post SM, Zhang N, Coombes K and Kornblau SM: p53 pathway dysfunction is highly prevalent in acute myeloid leukemia independent of TP53 mutational status. Leukemia 31: 1296-1305, 2017.

47. Ufkin ML, Peterson S, Yang X, Driscoll H, Duarte C and Sathyanarayana P: miR-125a regulates cell cycle, proliferation, and apoptosis by targeting the ErbB pathway in acute myeloid leukemia. Leuk Res 38: 402-410, 2014.

48. Curran E, Corrales L and Kline J: Targeting the innate immune system as immunotherapy for acute myeloid leukemia. Front Oncol 5: 83, 2015.

49. Takam Kamga P, Bassi G, Cassaro A, Midolo M, Di Trapani M, Gatti A, Carusone R, Resci F, Perbellini O, Gottardi M, et al: Notch signalling drives bone marrow stromal cell-mediated chemoresistance in acute myeloid leukemia. Oncotarget 7: 21713-21727, 2016

50. Witkowski MT, Cimmino L, Hu Y, Trimarchi T, Tagoh H, McKenzie MD, Best SA, Tuohey L, Willson TA, Nutt SL, et al: Activated Notch counteracts Ikaros tumor suppression in mouse and human T-cell acute lymphoblastic leukemia. Leukemia 29: $1301-1311,2015$

This work is licensed under a Creative Commons Attribution-NonCommercial-NoDerivatives 4.0 International (CC BY-NC-ND 4.0) License. 